\title{
Relação das teses defendidas na Faculdade de Direito em 86
}

\section{DISSERTAÇŌES E TESES}

1. Área: Direito Processual Autor: Jorge Luiz de Almeida

Dissertação: "EFICÁCIA DO JULGADO PENAL SOBRE O INTERESSE CIVIL"

Orientador: Cândido Rangel Dinamarco

Defendida em: 01/4/1986

2. Área: Direito Processual Autor: José Raul Gavião de Almeida Dissertação: "DA LEGITIMIDADE NA AÇÃO CIVIL PÚBLICA" Orientador: Cândido Rangel Dinamarco Defendida em: 02/4/1986

3. Área: Direito Civil

Autor: José Roberto Pacheco Di Francesco

Dissertação: "DA OBRIGAÇĀO ALIMENTAR ENTRE PARENTES" Orientador: Antonio Junqueira de Azevedo

Defendida em: 09/4/1986

4. Área: Direito Processual Autor: Nelson Hanada

Tese: “AS AÇŌES DE DEPÓSITO NO SISTEMA PROCESSUAL BRASILEIRO"

Orientador: Cândido Rangel Dinamarco

Defendida em: $14 / 4 / 1986$

5. Área: Direito do Estado Autor: Anna Britto da Rocha Acker Dissertação: "PODER NORMATIVO E REGIME DEMOCRÁTICO" Orientador: Dalmo de Abreu Dallari Defendida em: 18/4/1986 
6. Área: Direito Processual

Autor: José Rogério Cruz e Tucci

Dissertação: "DA SUPLICATIO AO AGRAVO ORDINÁRIO"

Defendida em: 23/5/1986

7. Área: Direito do Estado

Autor: Ronaldo Lindimar José Marton

Tese: "O REGULAMENTO E OS ATOS NORMATIVOS INFRAREGULAMENTARES NA PRÁTICA DA ADMINISTRAÇÃO"

Orientador: Antonio Carlos de Araújo Cintra

Defendida em: 27/5/1986

8. Área: Direito Econômico e Financeiro

Autor: Mário Jorge Góis Lopes

Tese: "REGIONALISMO E REGIONALIZAÇÃO ECONÔMICA DO ORDENAMENTO JURÍDICO"

Orientador: Eros Roberto Grau

Defendida em: 09/6/1986

9. Área: Direito Processual

Autor: José Carlos Gonçaives Xavier de Aquino

Dissertação: "PROVA TESTEMUNHAL NO PROCESSO PENAL"

Orientador: Rogério Lauria Tucci

Defendida em: 30/6/1986

10. Área: Filosofia e Teoria Geral do Direito

Autor: Clareci Mezzomo

Dissertação: "CONTROLE SOCIAL PELA SANÇÃO"

Orientador: Tércio Sampaio Ferraz Junior

Defendida em: $11 / 9 / 1986$

11. Área: Direito Internacional

Autor: Paulo Borba Casella

Tese: "APODERAMENTO ILÍCITO DE AERONAVES"

Orientador: Irineu Strenger

Defendida em: 12/9/1986

12. Área: Direito Civil

Autor: José Carlos Rocha de Carvalho

Tese: "HONRA E VERDADE NA INFORMAÇĀO"

Orientador: Walter Moraes

Defendida em: 17/9/1986 
13. Área: Direito Civil

Autor: Renata Helena Petri Gobbet

Dissertação: “ASPECTOS DOUTRINÁRIOS DA INVALIDADE

DE NEGÓCIO JURÍDICO NO DIREITO PRIVADO"

Orientador: Antonio Junqueira de Azevedo

Defendida em: 07/10/1986

14. Área: Direito do Estado

Autor: Alaor Caffé Alves

Tese: "ESTADO, APARÊNCIA E REALIDADE"

Orientador: Dalmo de Abreu Dallari

Defendida em: 09/10/1986

19. Área: Direito Comercial

Autor: Mauro Rodrigues Penteado

Tese: "AUMENTO DE CAPITAL MEDIANTE SUBSCRIÇÃO DE AÇÕES NO DIREITO BRASILEIRO"

Orientador: Luiz Gastāo Paes de Barros Leães

Defendida em: 24/10/1986

20. Área: Direito Civil

Autor: José Luiz Gavião de Almeida

Dissertação: "FILIAÇÃO INCESTUOSA - RECONHECIMENTO"

Orientador: Alvaro Villaça Azevedo

Defendida em: 29/10/1986

21. Área: Direito Comercial

Autor: Marcos Paulo de Almeida Salles

Dissertação: "UMA COṆTRIBUUIÇĀO À ANÁLISE DOS

$$
\text { DEBÊNTURES" }
$$

Orientador: Mauro Brandão Lopes

Defendida em: 31/10/1986

22. Área: Direito Civil

Autor: Ivani Martins Ferreira Giuliani

Dissertação: "CASAMEN1'O INEXISTENTE"

Orientador: Walter Moraes

Defendida em: $3 / 11 / 1986$ 
23. Área: Direito Comercial

Autor: Edmur de Andrade Nunes Pereira Neto

Dissertação: "ASPECTOS JURÍDICOS DA EMISSÃO DE

DEBÊNTURES CONVERSÍVEIS EM AÇÕES"

Orientador: Mauro Brandāo Lopes

Defendida em: $6 / 11 / 1986$

24. Área: Direito Civil

Autor: Daisy Gogliano

Tese: "O DIREITO AO TRANSPLANTE DE ÓRGÃOS E TECIDOS"

Orientador: Rubens Limongi França

Defendida em: $18 / 11 / 1986$

25. Área: Direito Internacional

Autor: Regina Barbara Nigro Mazon

Dissertação: "FREI FRANCISCO DE VITÓRIA: SUA CONTRIBUIÇÃO

AO CONCERNENTE À "RELECCIO DE INDIS"

E "RELECCIO DE JURE BELLI"

Orientador: Vicente Marotta Rangel

Defendida em: $19 / 11 / 1986$

26. Área: Direito Civil

Autor: Eugênio Facchini Neto

Dissertação: "RESPONSABILIDADE CIVIL PELO FATO DE OUTREM"

Orientador: Yussef Said Cahaii

Defendida em: 28/11/1986

27. Área: Direito do Estado

Autor: Norberto Guarinello

Dissertação: "DO DECRETO-LEI"

Orientador: José Cretella Junior

Defendida em: $1 / 12 / 1986$

28. Área: Direito Internacional

Autor: Wanda Vilardo de Mello

Dissertação: "REGIME JURÍDICO DAS ILHAS OCEÂNICAS

BRASILEIRAS"

Orientador: Vicente Marotta Rangel

Defendida em: 02/12/1986 
29. Área: Direito Penal

Autor: Augusto Martinez Perez

Dissertação: "REABILITAÇÃO PENAL NO DIREITO BRASILEIRO"

Orientador: Paulo José da Costa Junior

Defendida em: 05/12/1986

30. Área: Direito Civil

Autor: Jaira Grandisoli Parreira

Dissertação: "ASPECTOS LEGAIS DA ESTERILIZAÇÃO

VOLUNTÁRIA DO HOMEM E DA MULHER"

Orientador: Alvaro Villaça Azevedo

Defendida em: 05/12/1986

31. Área: Direito Civil

Autor: Helio Borghi

Dissertação: “A LESÃO NO ORDENAMENTO JURÍDICO BRASILEIRO"

Orientador: Fabio Maria de Mattia

Defendida em: 19/12/1986

32. Área: Direito Econômico e Financeiro

Autor: Geraldo Facó Vidigal

Tese: "MACROINTERESSES-UM TEMA DE DIREITO ECONÔMICO" Orientador: Fábio Nusdeo

Defendida em: 04/6/1987

33. Área: Direito do Estado

Autor: Dirceo Torrecillas Ramos

Dissertação: "TRANSIÇÃO CONSTITUCIONAL ESPANHOLA (1975-1978)"

Orientador: Manoel Gonçalves Ferreira Filho

Defendida em: $12 / 6 / 1987$ 